to/distance from hospital, using negative binomial regressions. In the other model the number of healthcare contacts observed during the follow-up period will be compared with the number expected to have occurred, based on pre injury healthcare contacts, to calculate standardised utilisation ratios. In both models general practice and inpatient data will be used to identify co-morbidities.

Results Analysis is ongoing and results will be presented at the conference.

Conclusion This study presents an enhanced approach to determining the extent of excess HSU and medical costs following injury, and will contribute to improved measurement of the burden of injury.

\title{
0590 COMPARISON OF DIFFERENT MODELS TO ASSESS THE MEDICAL COSTS OF INJURY
}

S Macey*, R A Lyons, C Phillips, S Brophy Correspondence: Swansea University, School of Medicine, Grove Building, Singleton Park, Swansea, Wales SA2 8PP, UK

10.1136/ip.2010.029215.590

Aim To compare different models of excess health service utilisation (HSU) to produce enhanced estimates of the short and medium term direct medical costs of injuries.

Method A retrospective and prospective anonymised electronic population cohort, encompassing all residents of Swansea, Wales, UK ( $\mathrm{n}=227$ 100). The Secure Anonymised Information Linkage (SAIL) system is being used to anonymously track injured and uninjured individuals through multiple healthcare databases over a 3 year period. Excess HSU within emergency department, inpatient and outpatient sectors will be determined using two different models. In one the HSU postinjury will be counted and subsequently adjusted for potential confounders, including age, gender, deprivation status, preinjury healthcare utilisation, co-morbidities and access 